\title{
Properties of Primary Cosmic Ray Protons, Helium, Carbon and Oxygen Nuclei Measured with the Alpha Magnetic Spectrometer on the International Space Station
}

Yi Jia*†

Massachusetts Institute of Technology

E-mail: jiay@mit.edu

\begin{abstract}
We report on the precision high statistics measurements of primary cosmic ray protons, helium, carbon and oxygen fluxes by Alpha Magnetic Spectrometer (AMS) in the rigidity (momentum per unit charge) range from $2 \mathrm{GV}$ to $3 \mathrm{TV}$. These measurements are based on 1 billion protons, 125 million helium, 14 million carbon and 12 million oxygen nuclei collected by AMS during the first seven years of operation aboard the International Space Station. Precision measurements from AMS reveal many unexpected properties of cosmic rays.
\end{abstract}

XXIX International Symposium on Lepton Photon Interactions at High Energies - LeptonPhoton2019 August 5-10, 2019

Toronto, Canada

*Speaker.

$\dagger$ on behalf of the AMS Collaboration. 


\section{Introduction}

Traditionally, there are two prominent classes of charged cosmic rays. Primary cosmic rays $(\mathrm{p}, \mathrm{He}, \mathrm{C}, \mathrm{O}, \ldots$ ) are produced at their sources and travel through space, and are directly detected by AMS. They carry information on their source and the history of their travel. Secondary cosmic rays $(\mathrm{Li}, \mathrm{Be}, \mathrm{B}, \ldots)$ are mainly produced in the collisions of primary cosmic rays. They carry information on the history of their travel, and the properties of the interstellar medium (ISM). In addition, the secondary to primary flux ratios, such as the $\mathrm{B} / \mathrm{C}$ ratio, measure the average amount of interstellar material traversed by cosmic rays, and provide important input in the propagation models [1].

The precision measurements from AMS have revealed many unexpected properties of cosmic nuclei. Primary cosmic ray helium, carbon, and oxygen were found to have an identical rigidity dependence above $60 \mathrm{GV}$ [2]. Secondary cosmic ray lithium, beryllium, and boron also show identical rigidity dependence above $30 \mathrm{GV}$, but their behavior is distinctly different compared to the primary cosmic rays [3]. All measured AMS nuclei spectra $(\mathrm{Z} \leq 8)$ show a deviation from a single power law above $\sim 200 \mathrm{GV}[2,3,4,5]$. With large acceptance and long exposure time, AMS will be able to measure the fluxes of cosmic nuclei up to iron and beyond.

\section{The AMS Detector}

The AMS detector is a general purpose particle physics detector in space. The schematic of the AMS detector is shown in Fig. 1. The key elements used in these measurements are the permanent magnet [6], the silicon tracker [7], and the four planes of time of flight (TOF) scintillation counters [8].

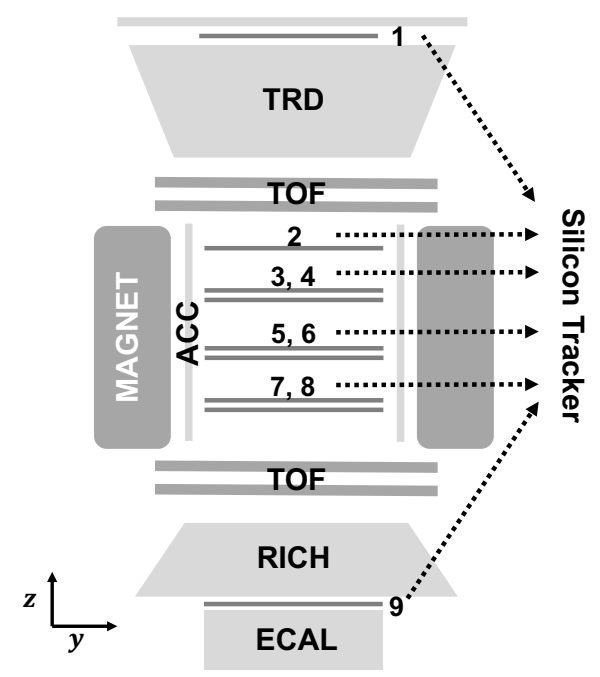

Figure 1: Schematic of the AMS detector. It contains a Transition Radiation Detector (TRD), nine planes of silicon tracker, surrounded by an array of 16 Anti-Coincidence Counters (ACC), an Electromagnetic Calorimeter (ECAL), a Time of Flight detector (TOF) with four planes of scintillating paddles and a Ring Image Cherenkov (RICH) detector. 
As seen in Fig. 1, the AMS silicon tracker has nine layers. Tracker L1 and L9, separated by a distance of 3 meters, are on top of the TRD and the ECAL, respectively. Tracker L2 is above the magnet, and six layers (L3 to L8) are within the bore of the magnet. L2 to L8 constitute the inner tracker.

Each layer of the tracker provides an independent measurement of the charge $\mathrm{Z}$ with a resolution of $\Delta \mathrm{Z} / \mathrm{Z}=9 \%$ for helium, $5 \%$ for carbon, and $4 \%$ for oxygen. Overall, the inner tracker has a resolution of $\Delta \mathrm{Z} / \mathrm{Z}=3.5 \%$ for helium, $2 \%$ for carbon, and $1.5 \%$ for oxygen. The spatial resolution in each tracker layer is $6.5 \mu \mathrm{m}$ in the bending direction for helium, $5.1 \mu \mathrm{m}$ for carbon, and 6.3 $\mu \mathrm{m}$ for oxygen [9]. Together, the tracker and the magnet measure the rigidity $\mathrm{R}$ of charged cosmic rays, with a maximum detectable rigidity (MDR) of 3.2 TV for helium, 3.7 TV for carbon, and 3.4 TV for oxygen over the $3 \mathrm{~m}$ lever arm from L1 to L9.

Two of the TOF planes are located above the magnet (upper TOF) and two planes are below the magnet (lower TOF). The overall velocity $\left(\beta=\mathrm{v} / \mathrm{c}\right.$ ) resolution has been measured to be $\Delta \beta / \beta^{2}$ $=0.02$ for helium and $\Delta \beta / \beta^{2}=0.01$ for carbon and oxygen nuclei. This discriminates between upward- and downward-going particles. The pulse heights of the two upper planes are combined to provide an independent measurement of the charge with an accuracy $\Delta \mathrm{Z} / \mathrm{Z}=4 \%$ for helium, $3 \%$ for carbon, and $2 \%$ for oxygen. The pulse heights from the two lower planes are combined to provide another independent charge measurement with the same accuracy.

\section{Update Results on Primary Cosmic Rays (p, He, C, O)}

Our previous publication on protons in 2015 [4] is based on 300 million events. The previous publication on $\mathrm{He}, \mathrm{C}$, and $\mathrm{O}$ [2] is based on 90 million helium, 8.4 million carbon, and 7.0 million oxygen nuclei collected during the first five years of operation. Since then, we have completed the analysis with the data sample corresponding to seven years of operation.

The latest AMS measurements of primary cosmic rays are based on 1 billion protons, 125 million / nuclei, 14 million carbon nuclei and 12 million oxygen nuclei collected from May 2011 to May 2018. Fig 2 shows the updated AMS proton flux together with the earlier measurements. Our latest result is in agreement with our published result but has smaller errors.

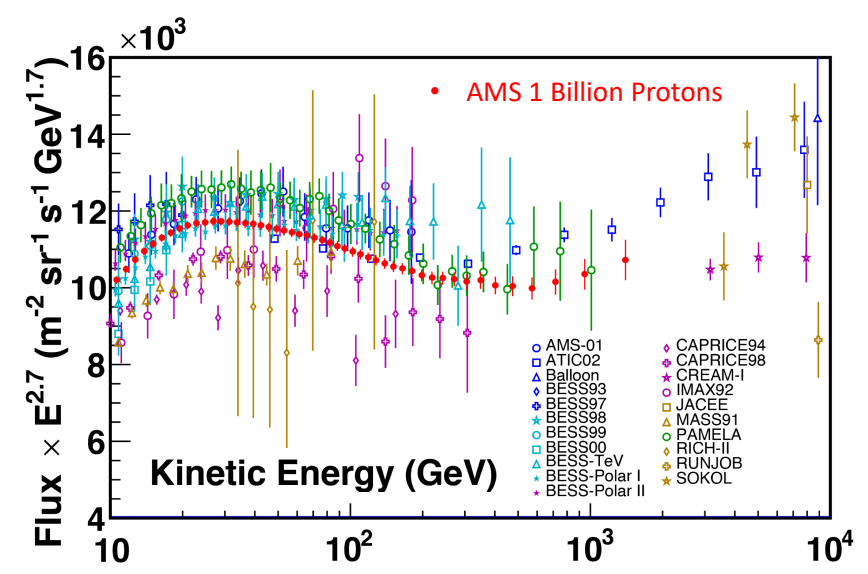

Figure 2: Latest AMS proton flux multiplied by $E^{2.7}$ together with previous measurements. Please refer to upcoming AMS publication on Physics Reports [10]. 
Figure 3 shows the updated AMS helium flux together with the earlier measurements. Again, the latest result is in agreement with our published result but has smaller errors.

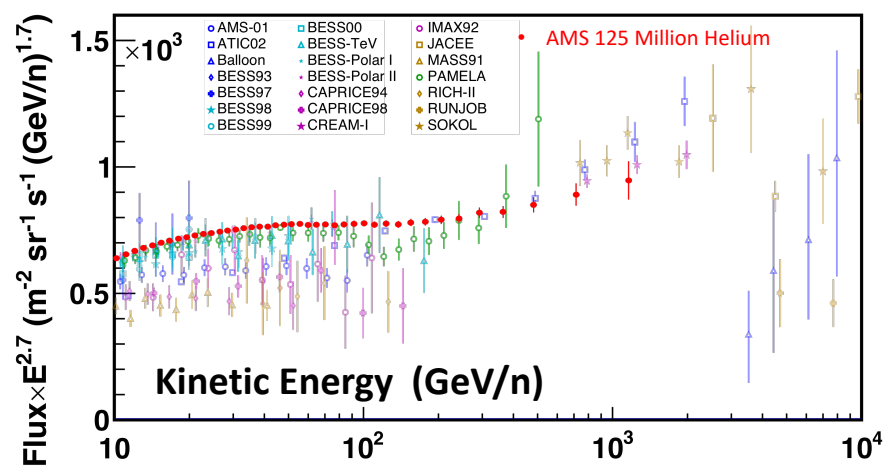

Figure 3: Latest AMS helium flux multiplied by $E^{2.7}$ together with previous measurements. Please refer to upcoming AMS publication on Physics Reports [10].

Figure 4 shows the updated AMS fluxes of primary cosmic rays $\mathrm{He}, \mathrm{C}$, and $\mathrm{O}$ as function of rigidity multiplied by $\tilde{R}^{2.7} . \tilde{R}$ is the spectrally weighted mean rigidity for a flux proportional to $R^{2.7}$. Again, these results are in agreement with our published result but have smaller errors. As seen, above $60 \mathrm{GV}$ all three spectra have identical rigidity dependence. In particular, they all deviate from a single power law and harden from $\sim 200 \mathrm{GV}$.

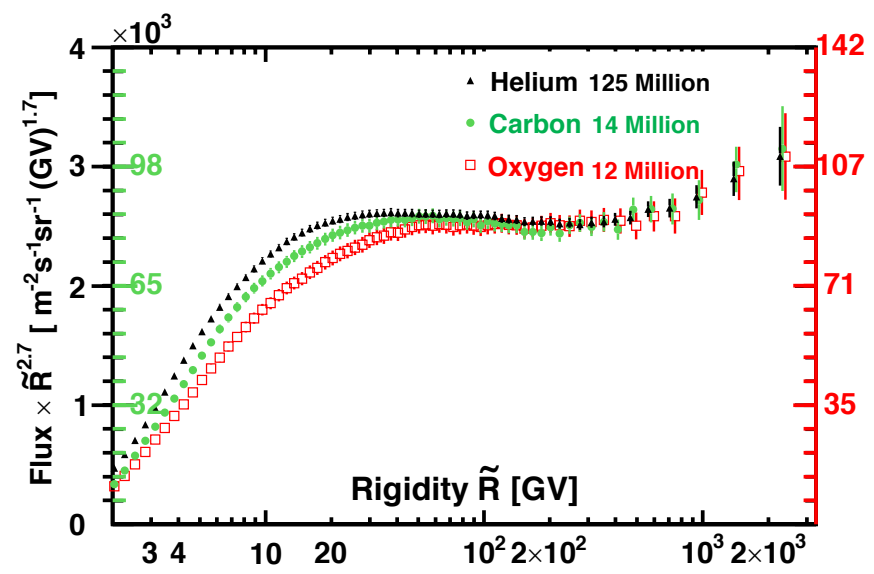

Figure 4: Latest AMS helium (black, left axis), carbon (green, left axis), and oxygen (red, right axis) flux as a function of rigidity multiplied by $\tilde{R}^{2.7}$ together with previous measurements. For clarity, horizontal positions of the helium and oxygen data points above $400 \mathrm{GV}$ are displaced with respect to the carbon. Please refer to upcoming AMS publication on Physics Reports [10].

Figure 5 [3] shows the comparison of the rigidity dependences of the AMS secondary cosmic ray fluxes with the primary cosmic ray fluxes. The amplitudes of these fluxes have been rescaled for display purposes. As seen, an identical rigidity dependence has also been observed in secondary cosmic rays $\mathrm{Li}, \mathrm{Be}$, and $\mathrm{B}$, but the secondary cosmic ray rigidity dependence is distinctly different from the rigidity dependence of primary cosmic rays. 


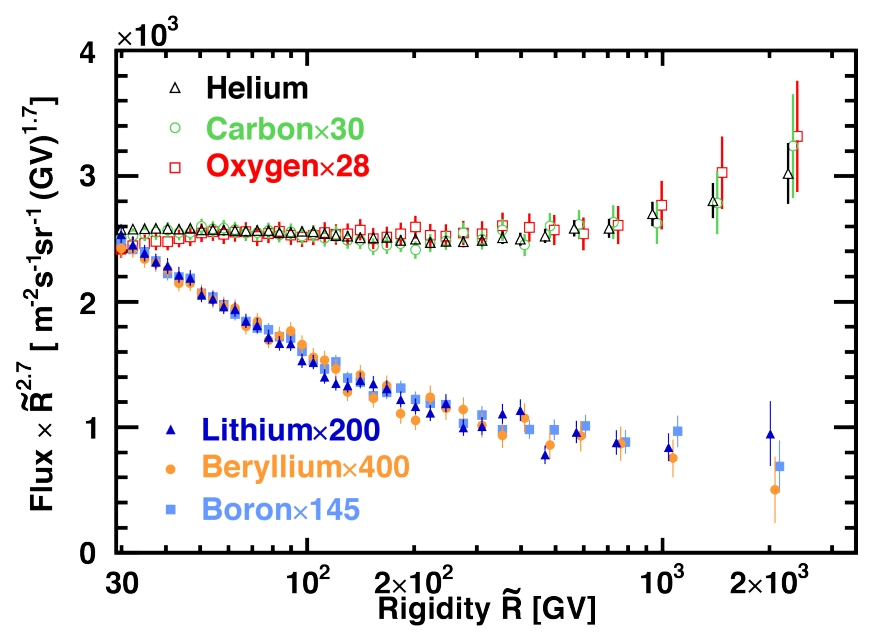

Figure 5: Comparison of the AMS secondary cosmic ray fluxes with the primary cosmic ray fluxes multiplied by $\tilde{R}^{2.7}$ with their total error as a function of rigidity above $30 \mathrm{GV}$. For display purposes only, the C, $\mathrm{O}, \mathrm{Li}, \mathrm{Be}$, and $\mathrm{B}$ fluxes were rescaled as indicated. For clarity, the $\mathrm{He}, \mathrm{O}, \mathrm{Li}$, and B data points above 400 $\mathrm{GV}$ are displaced horizontally.

\section{Conclusions}

The latest AMS results on primary cosmic rays (p, He, C, O) based on data collected during the first seven years of operation are in agreement with our previous published results. They all deviate from a single power law above $\sim 200 \mathrm{GV}$. Identical rigidity dependences are observed for both primary cosmic rays $(\mathrm{He}, \mathrm{C}$, and $\mathrm{O}$ ) and secondary cosmic rays $(\mathrm{Li}, \mathrm{Be}$, and $\mathrm{B})$, but they are distinctly different.

\section{References}

[1] I. A. Grenier, J. H. Black, and A. W. Strong, Annu. Rev. Astron. Astrophys. 53, 199 (2015); P. Blasi, Astron. Astrophys. Rev. 21, 70 (2013); A. W. Strong, I. V. Moskalenko, and V. S. Ptuskin, Annu. Rev. Nucl. Part. Sci. 57, 285 (2007); A. Castellina and F. Donato, Astrop. Phys. 24, 1-2 (2005).

[2] M. Aguilar et al., Phys. Rev. Lett. 119, 251101 (2017).

[3] M. Aguilar et al., Phys. Rev. Lett. 120, 021101 (2018).

[4] M. Aguilar et al., Phys. Rev. Lett. 114, 534871 (2015).

[5] M. Aguilar et al., Phys. Rev. Lett. 121, 051103 (2018).

[6] K. Lübelsmeyer et al., Nucl. Instrum. Methods Phys. Res., Sect. A 654, 639 (2011).

[7] B. Alpat et al., Nucl. Instrum. Methods Phys. Res., Sect. A 613, 207 (2010).

[8] V. Bindi et al., Nucl. Instrum. Methods Phys. Res., Sect. A 743, 22 (2014).

[9] G. Ambrosi, V. Choutko, C. Delgado, A. Oliva, Q. Yan, and Y. Li, Nucl. Instrum. Methods Phys. Res., Sect. A 869, 29 (2017).

[10] AMS Collabration, The Alpha Magnetic Spectrometer (AMS) on the International Space Station: Part II, (to be published in Physics Reports). 